\title{
Repouso de Três Horas Não Aumenta Complicações Após Cateterismo Cardíaco Diagnóstico com Introdutor Arterial 6 F: Ensaio Clínico Randomizado*
}

\author{
Vanety Silva Rocha', Graziella Aliti2,3, Maria Antonieta Moraes², Eneida Rejane Rabelo ${ }^{2,3,4}$
}

\section{RESUMO}

Introdução: Atualmente o tempo de permanência ideal no leito após cateterismo cardíaco diagnóstico não obedece a um consenso. Sua redução, dentro dos limites de segurança, aperfeiçoaria a utilização dos recursos existentes diante da demanda crescente de exames, além de melhorar o conforto dos pacientes. O objetivo deste estudo foi avaliar a segurança na redução do tempo de repouso no leito, de seis para três horas, após cateterismo cardíaco diagnóstico com introdutor arterial 6 F. Método: Ensaio clínico randomizado, realizado em laboratório de cardiologia intervencionista em Santa Maria (RS), entre agosto de 2007 e novembro de 2008. Foram incluídos pacientes submetidos a cateterismo cardíaco diagnóstico, com idade $\geq 18$ anos, de ambos os sexos, excluindo-se pacientes em uso de anticoagulantes, com obesidade mórbida, e história de discrasias sanguíneas, doenças da aorta ou com hipertensão arterial grave não-controlada. Os pacientes foram divididos em grupo intervenção, que deambulou três horas após remoção da bainha, e grupo controle, que deambulou seis horas após repouso no leito. Todos foram monitorados, a cada hora, pela equipe de enfermagem, e 24, 48 e 72 horas após a alta hospitalar, por meio de contato telefônico. Resultados: No total, foram incluídos 406 pacientes (200 no grupo intervenção e 206 no grupo controle), com média de idade de $64 \pm 9,4$ anos, 47,3\% do sexo feminino e $17 \%$ de diabéticos. Sangramento ocorreu em $1(0,5 \%)$ caso no grupo intervenção e em $4(1,9 \%)$ no grupo controle; ocorreram $3(1,5 \%)$ casos de hematoma no grupo intervenção e 4 (1,9\%), no grupo controle; 4 (2\%) pacientes apresentaram reação vasovagal no grupo intervenção e $7(3,4 \%)$, no grupo controle. Não houve diferença entre os grupos para qualquer das comparações. Conclusões: A redução do tempo de repouso para três

* Monografia do Curso de Especialização de Enfermagem em Cardiologia do Instituto de Cardiologia do Rio Grande do Sul/Fundação Universitária de Cardiologia - Porto Alegre, RS, Brasil.

1 Clínica de Hemodinâmica Hemocor - Santa Maria, RS, Brasil.

2 Pós-graduação Lato Sensu em Enfermagem em Cardiologia do Instituto de Cardiologia - Fundação Universitária de Cardiologia Porto Alegre, RS, Brasil.

${ }^{3}$ Hospital de Clínicas de Porto Alegre (HCPA) - Porto Alegre, RS, Brasil.

${ }^{4}$ Escola de Enfermagem da Universidade Federal do Rio Grande do Sul - Porto Alegre, RS, Brasil.

Correspondência: Eneida R. Rabelo. Rua Princesa Isabel, 370 Santana - Porto Alegre, RS, Brasil - CEP 90620-000

E-mail: editoracao-pc@cardiologia.org.br; rabelo@portoweb.com.br Recebido em: 29/9/2009 - Aceito em: 1\%/12/2009

\section{ABSTRACT}

\author{
Three-Hour Rest Period After Cardiac \\ Catheterization with a 6 F Sheath Does Not \\ Increase Complications: A Randomized Clinical Trial
}

Background: Currently, bed rest time after diagnostic cardiac catheterization does not follow a consensus. Reducing it, within safety thresholds, would optimize the use of the existing resources in face of the increasing demand of these exams and would also improve patient comfort. The objective of this study was to evaluate the safety of reducing bed rest time from six to three hours after diagnostic cardiac catheterization with a $6 \mathrm{~F}$ arterial sheath. Method: Randomized clinical trial carried out at an Interventional Cardiology laboratory in Santa Maria, Rio Grande do Sul, Brazil, from August 2007 to November 2008. Male and female patients undergoing diagnostic catheterization aged $\geq 18$ years were included and patients on oral anticoagulants, with morbid obesity, history of bleeding, aortic diseases or non-controlled severe hypertension were excluded. Patients were divided into an intervention group (IG), with ambulation three hours after sheath removal, and a control group (CG), with ambulation after six hours of bed rest. They were all monitored hourly by the nursing team and 24, 48 and 72 hours after discharge by telephone contact. Results: Overall, 406 patients were included (200 in the IG and 206 in the CG), mean age was $64 \pm 9.4$ years, $47.3 \%$ were women and $17 \%$ were diabetic. There was 1 case of bleeding $(0.5 \%)$ in the IG and $4(1.9 \%)$ in the CG; there were $3(1.5 \%)$ cases of hematoma in the IG and $4(1.9 \%)$ in the CG; $4(2 \%)$ patients presented a vasovagal reaction in the IG and $7(3.4 \%)$ in the CG. There were no statistical differences between the two groups for any of the comparisons. Conclusions: The reduction of bed rest to three 
Rocha VS, et al. Repouso de Três Horas Não Aumenta Complicações Após Cateterismo Cardíaco Diagnóstico com Introdutor Arterial 6 F: Ensaio Clínico Randomizado. Rev Bras Cardiol Invas. 2009;17(4):512-7.

horas não aumentou as complicações nos pacientes que realizaram cateterismo cardíaco diagnóstico com introdutor arterial $6 \mathrm{~F}$, mostrando-se segura quando comparada ao repouso de seis horas.

DESCRITORES: Doença das coronárias/terapia. Deambulação precoce. Cuidados de enfermagem. Cateterismo cardíaco. Procedimentos cirúrgicos ambulatórios. Repouso em cama. Ensaio clínico controlado aleatório.

0 período de repouso no leito após cateterismo cardíaco tem sido objeto de investigações ao longo dos últimos anos ${ }^{1,2}$. De acordo com a literatura, ainda não foi possível chegar a um consenso a respeito do tempo ideal de repouso após a retirada da bainha femoral. Resultados de estudos que reduziram de seis para quatro horas, e de quatro para duas horas, o tempo de repouso de pacientes submetidos a cateterismo cardíaco com introdutores $6 \mathrm{~F}$ não identificaram maior número de complicações no grupo que deambulou mais precocemente ${ }^{3,4}$. Outro estudo com pacientes submetidos a cateterismo cardíaco diagnóstico com introdutores $5 \mathrm{~F}$ reduziu para uma hora o tempo de repouso, sem aumentar as complicações ${ }^{5}$.

Embora a literatura indique que a deambulação precoce seja segura e factível, não foram encontrados, até o momento, estudos semelhantes conduzidos no Brasil. Dentro desse contexto, com o objetivo de aperfeiçoar os recursos existentes quanto à demanda crescente de exames, de reduzir os custos hospitalares e de melhorar o conforto aos pacientes, delineou-se um ensaio clínico randomizado para avaliar a segurança na redução do tempo de repouso no leito de seis para três horas após cateterismo cardíaco diagnóstico com introdutor $6 \mathrm{~F}$.

\section{MÉTODO}

Ensaio clínico randomizado que incluiu pacientes submetidos a cateterismo cardíaco eletivo, por via femoral, utilizando cateteres e bainha $6 \mathrm{~F}$, no período de agosto de 2007 a novembro de 2008, em um laboratório de hemodinâmica em Santa Maria (RS). Selecionaram-se pacientes $\geq 18$ anos, de ambos os sexos, submetidos a cateterismo cardíaco eletivo. Excluíram-se os pacientes em uso de anticoagulantes (cumarínicos e heparina) e aqueles com obesidade mórbida (índice de massa corporal $\geq 40 \mathrm{~kg} / \mathrm{m}^{2}$ ), história de discrasias sanguíneas, doenças da aorta ou hipertensão arterial grave não-controlada (sistólica $\geq 180 \mathrm{mmHg}$, diastólica $\geq 110 \mathrm{mmHg}$ ). Este estudo foi aprovado pelo Comitê de Ética em Pesquisa da instituição, sob número 4019/07. Os pacientes foram orientados sobre os objetivos do presente estudo, sendo incluídos após terem lido e assinado o Termo de Consentimento Livre Esclarecido. hours did not increase complications in patients undergoing diagnostic cardiac catheterization with a $6 \mathrm{~F}$ arterial sheath, and proved to be safe when compared to the sixhour rest period.

DESCRIPTORS: Coronary disease/therapy. Early ambulation. Nursing care. Heart catheterization. Ambulatory surgical procedures. Bed rest. Randomized controlled trial.

\section{Protocolo do estudo}

As secretárias da clínica agendaram o comparecimento dos pacientes para a realização de cateterismo de segunda a sexta-feira. Na data marcada, os pacientes foram avaliados e os que preenchessem os critérios de inclusão eram encaminhados para uma sala de preparo, onde uma enfermeira os aguardava para realizar o convite a participar da pesquisa, explicando o estudo. Após entender os objetivos do estudo e concordar com a participação, os pacientes assinavam o Termo de Consentimento Livre Esclarecido. Após esse procedimento, os pacientes foram inseridos, pela secretária, em uma lista de randomização, gerada previamente pelo programa Random. A pesquisadora do estudo era cega para essa randomização. O protocolo de rotinas foi seguido normalmente até o final do cateterismo cardíaco eletivo. Ao término, o paciente era encaminhado da sala de exame para a sala de recuperação, onde, no leito, a equipe de enfermagem treinada removia a bainha $6 \mathrm{~F}$, realizando compressão manual por 20 minutos, seguida da colocação de peso de $2 \mathrm{~kg}$ no local da punção, até o término do repouso. $\mathrm{O}$ paciente era orientado a manter o membro puncionado imobilizado. Na terceira hora de repouso a equipe de enfermagem entrava em contato com a secretária do laboratório por telefone interno, para ser informada sobre a randomização do paciente. Ambos os grupos recebiam orientações por escrito sobre os cuidados pós-cateterismo, sendo avisados de que a enfermeira entraria em contato telefônico para acompanhamento após a alta. Para cada paciente foi preenchida uma ficha clínica pela equipe de enfermagem, com dados referentes à deambulação e ao quadro clínico. No contato telefônico, realizado pela enfermeira em 24, 48 e 72 horas após a alta, era questionado o aspecto do local da punção (presença de edema, sangramento, hematoma ou outra reação). Todas as informações foram registradas na ficha clínica.

As complicações vasculares foram definidas como: 1) hematoma no local da punção arterial (pequeno, $<1 \mathrm{~cm}$; médio, $1 \mathrm{~cm}$ a $5 \mathrm{~cm}$; e grande, $>5 \mathrm{~cm}$ ); 2) sangramento (menor, sem instabilidade hemodinâmica; maior, com instabilidade hemodinâmica); 3) correção cirúrgica da complicação vascular (hematoma retroperitoneal, pseudoaneurisma ou formação de fístu- 
Rocha VS, et al. Repouso de Três Horas Não Aumenta Complicações Após Cateterismo Cardíaco Diagnóstico com Introdutor Arterial 6 F: Ensaio Clínico Randomizado. Rev Bras Cardiol Invas. 2009;17(4):512-7.

la arteriovenosa); 4) reação vasovagal (leve, vertigem; moderada, hipertensão e bradicardia; e grave, tontura, hipotensão e bradicardia, que necessitasse da infusão de volume ou medicação).

Os pacientes alocados para o grupo intervenção mantiveram repouso no leito por três horas, sendo, em seguida, estimulados a sentar no leito por 10 minutos e posteriormente deambular curtas distâncias, alternadas com momentos de repouso, sentados no leito, com cabeceira elevada a 45 graus, por mais três horas. Durante o tempo de repouso, o local da punção era observado de hora em hora pela equipe de enfermagem.

Os pacientes alocados para o grupo controle mantiveram repouso por seis horas. Depois desse período, eram estimulados a sentar no leito por 10 minutos, com cabeceira elevada em 45 graus, e, após, deambular por mais 10 minutos, recebendo alta a seguir. Também durante o tempo de repouso foi observado o local da punção de hora em hora pela equipe de enfermagem.

\section{Análise estatística}

As variáveis contínuas foram expressas como média \pm desvio padrão e analisadas pelo teste $t$ de Student. As variáveis categóricas foram expressas em frequências e porcentuais e comparadas com o teste de qui-quadrado de Pearson ou exato de Fischer. $\mathrm{P}<0,05$ foi considerado significante. Todos os dados foram analisados com o auxílio do programa estatístico Statistical Package for Social Sciences 15.0.

\section{RESULTADOS}

\section{População estudada}

No período de agosto de 2007 a novembro de 2008, 680 pacientes foram admitidos para cateterismo cardíaco eletivo, dos quais 406 que aceitaram participar do protocolo e que preencheram os critérios de inclusão e exclusão foram randomizados para dois grupos: 200 no grupo intervenção e 206 no grupo controle (Figura 1).

\section{Características demográficas e clínicas da população}

A média de idade dos 406 pacientes foi de $64 \pm 9,4$ anos, $47,3 \%$ eram do sexo feminino e $17 \%$ eram diabéticos. Não houve diferença na comparação de quaisquer das variáveis clínicas ou demográficas avaliadas entre os grupos (Tabela 1).

\section{Complicações pós-cateterismo cardíaco}

Hematoma foi observado em 3 pacientes do grupo intervenção, todos de pequeno tamanho, e em 4 pacientes do grupo controle (1 de médio e 3 de pequeno porte). Sangramento foi observado em 1 caso do grupo intervenção e em 4 do grupo controle, todos após o início da deambulação, de acordo com o protocolo estipulado para cada grupo. Esses sangramentos foram classificados como menores, requerendo apenas compressão manual adicional de $20 \mathrm{mi}-$ nutos. Ao término da compressão, manteve-se repouso adicional de mais seis horas. Reações vagais foram notadas em 4 pacientes do grupo intervenção, sendo leves em 3 pacientes e moderadas em 1 paciente. No grupo controle, a reação vasovagal foi classificada como leve em 6 pacientes e grave em 1 paciente. Esses dados estão apresentados, de forma resumida, na Tabela 2 .

Não foram verificadas complicações vasculares que necessitassem correção cirúrgica nos dois grupos.

\section{DISCUSSÃO}

Neste estudo, a redução do tempo de repouso no leito de seis para três horas, em pacientes submetidos a cateterismo cardíaco por via femoral com introdutores $6 \mathrm{~F}$, não aumentou a frequência de hematomas de via de acesso, sangramentos ou reações vasovagais, demonstrando a segurança dessa estratégia.

Os episódios de sangramento ocorreram assim que se iniciou a mobilização, após o tempo estipulado para cada grupo. Esses sangramentos foram classificados como menores, requerendo apenas compressão manual adicional de 20 minutos. Ao término da compressão, iniciou-se o repouso de mais seis horas, seguindo o protocolo da instituição.

Boztosun et al. ${ }^{6}$ avaliaram 1.446 pacientes submetidos a cateterismo cardíaco diagnóstico com introdutor arterial $6 \mathrm{~F}$, dos quais 1.226 (85\%) deambularam em duas horas e foram liberados, e o restante, que apresentou alguma intercorrência (dificuldade de acesso arterial, hipertensão não-controlada, compressão prolongada, etc.), permaneceu por duas a três horas adicionais em observação. Nenhum sangramento maior ou grande hematoma foi notado durante a observação hospitalar, à exceção de equimoses $(10 \%$ vs. $21 \%$, respectivamente, para o grupo com deambulação em duas horas e para o grupo com deambulação mais demorada) e pequenos hematomas (22\% vs. 9\%, respectivamente, para o grupo com deambulação em duas horas e para o grupo com deambulação mais demorada) após a alta, levando os autores a concluir que a deambulação precoce é segura e cursa com taxa aceitável de complicações hemorrágicas ${ }^{6}$.

A identificação de fatores de risco para complicações vasculares após cateterismo cardíaco pode levar à implementação de estratégias para a prevenção dessas complicações. Avaliação de mais de $11 \mathrm{mil}$ cateterismos e intervenções percutâneas identificou idade $>70$ anos, sexo feminino, insuficiência renal, intervenções percutâneas e introdutores venosos como fatores associados a complicações vasculares ${ }^{7}$. Em 


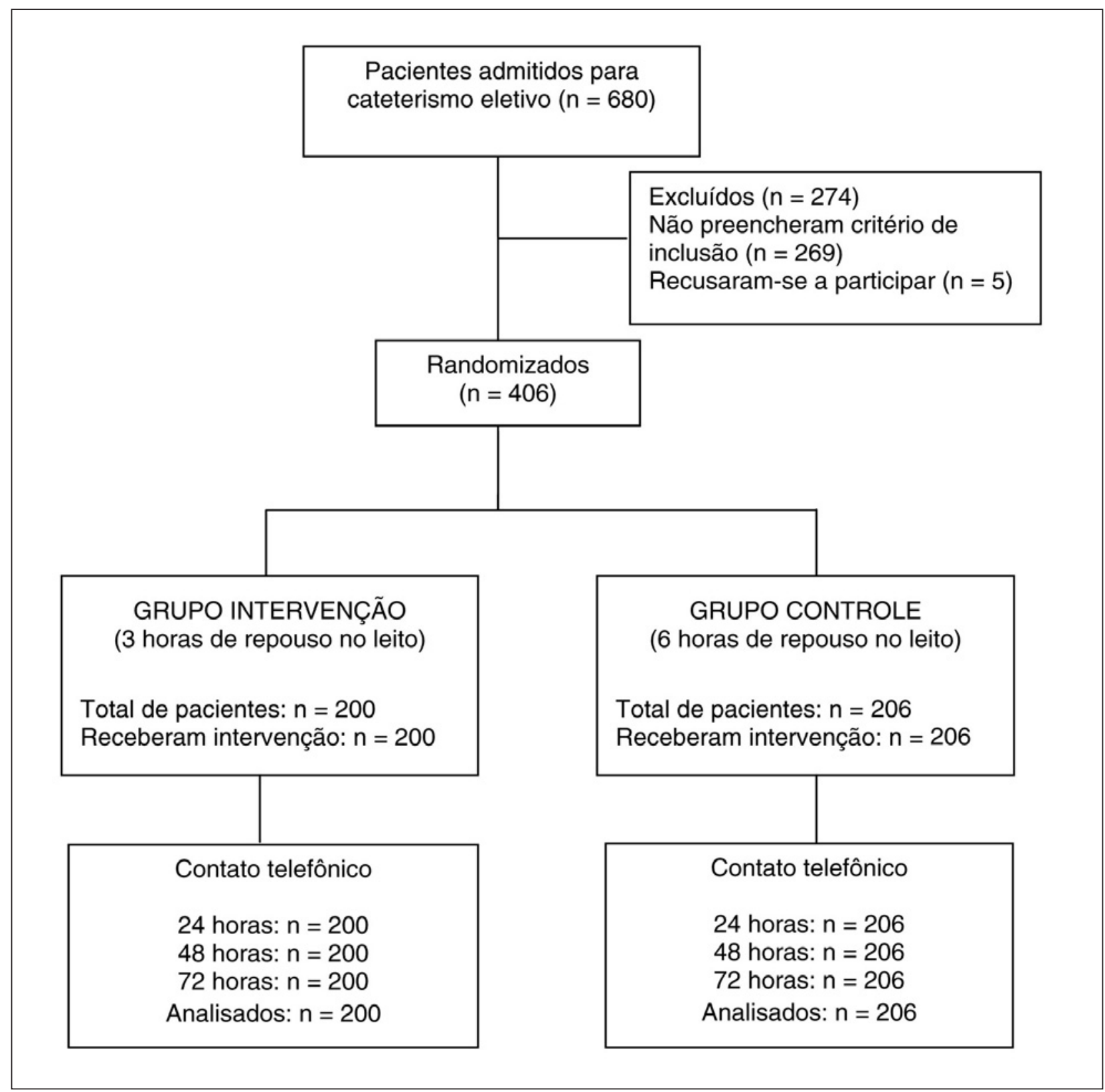

$\mathrm{n}=$ número de pacientes.

Figura 1 - Diagrama Consort.

nosso meio, o sexo feminino e a utilização de abciximab foram preditores de complicações vasculares após intervenções coronárias percutâneas, enquanto a utilização de dispositivos hemostáticos foi fator protetor $^{8}$. Análise das complicações vasculares em mulheres, no período de 1998 a 2005, mostrou que essas complicações decresceram ao longo do tempo, possivelmente em decorrência dos cuidados e da adoção de medidas de proteção, e, no final do acompanhamento, já não mostravam mais diferenças em relação ao sexo masculino ${ }^{9}$.
As reações vasovagais, outra das complicações analisadas neste estudo, ocorrem em 3,4\% a 13,9\% dos procedimentos coronários percutâneos, e fatores como presença de dor, lesão tecidual e estados emocionais alterados têm sido identificados como predisponentes ${ }^{10}$. O mal-estar gerado pelo decúbito dorsal e pela imobilidade, associado a dificuldade de micção, desconforto pélvico e ansiedade, são preditores da ocorrência da reação vasovagal durante a retirada da bainha arterial pós-intervenção coronária percutânea, e a redução do tempo de repouso nesse cenário pode reduzir essa complicação. 
Rocha VS, et al. Repouso de Três Horas Não Aumenta Complicações Após Cateterismo Cardíaco Diagnóstico com Introdutor Arterial 6 F: Ensaio Clínico Randomizado. Rev Bras Cardiol Invas. 2009;17(4):512-7.

TABELA 1

Características demográficas e clínicas

\begin{tabular}{lcccc}
\hline Características & $\begin{array}{c}\text { Todos } \\
(\mathbf{n}=\mathbf{4 0 6})\end{array}$ & $\begin{array}{c}\text { Grupo intervenção } \\
(\mathbf{n}=\mathbf{2 0 0})\end{array}$ & $\begin{array}{c}\text { Grupo controle } \\
(\mathbf{n}=\mathbf{2 0 6})\end{array}$ & $\mathbf{P}$ \\
\hline Idade, anos & $64 \pm 9,4$ & $63,5 \pm 9,5$ & $64,5 \pm 9,3$ & 0,32 \\
Sexo feminino, $\mathrm{n}(\%)$ & $192(47,3)$ & $101(50,5)$ & $91(44,2)$ & 0,20 \\
Peso, kg & $75 \pm 13,7$ & $75,2 \pm 13,8$ & $74,8 \pm 13,6$ & 0,76 \\
Altura, cm & $1,67 \pm 0,08$ & $1,67 \pm 0,09$ & $1,67 \pm 0,08$ & 0,39 \\
Índice de massa corporal, $\mathrm{kg} / \mathrm{m}^{2}$ & $27 \pm 4,3$ & $27,1 \pm 4,4$ & $27 \pm 4,1$ & 0,92 \\
Diabetes, $\mathrm{n}(\%)$ & $69(17)$ & $35(17,5)$ & $34(16,5)$ & 0,78 \\
$\quad$ Insulina, $\mathrm{n}(\%)$ & $7(1,72)$ & $4(2)$ & $3(1,5)$ & 0,27 \\
Hipertensão arterial sistêmica, $\mathrm{n}(\%)$ & $245(60,3)$ & $114(57)$ & $131(63,6)$ & 0,17 \\
Doença periférica vascular, $\mathrm{n}(\%)$ & $35(8,6)$ & $20(10)$ & $15(7,3)$ & 0,32 \\
Medicações em uso, $\mathrm{n}(\%)$ & & & & \\
$\quad$ Ácido acetilsalicílico & $226(55,7)$ & $108(54)$ & $25(12,1)$ & 0,50 \\
$\quad$ Clopidogrel & $49(12,1)$ & $24(12)$ & $3(1,5)$ & 0,96 \\
$\quad$ Ticlopidina & $5(1,2)$ & $2(1)$ & $128,7 \pm 14,8$ & 0,14 \\
PAS, mmHg & $127,6 \pm 15,2$ & $126,5 \pm 15,6$ & $79,8 \pm 8,6$ & 0,06 \\
PAD, mmHg & $78,8 \pm 8,7$ & $77,9 \pm 8,6$ & & \\
\hline
\end{tabular}

$\mathrm{n}=$ número de pacientes; $\mathrm{PAD}=$ pressão arterial diastólica; PAS = pressão arterial sistólica.

TABELA 2

Complicações pós-cateterismo cardíaco

\begin{tabular}{lcccc}
\hline Complicações & $\begin{array}{c}\text { Todos } \\
(\mathbf{n = 4 0 6})\end{array}$ & $\begin{array}{c}\text { Grupo intervenção } \\
(\mathbf{n}=\mathbf{2 0 0})\end{array}$ & $\begin{array}{c}\text { Grupo controle } \\
(\mathbf{n = ~ 2 0 6 )}\end{array}$ & $\mathbf{P}$ \\
\hline Hematoma, $\mathrm{n}(\%)$ & $7(1,7)$ & $3(1,5)$ & $4(1,9)$ & 0,28 \\
Sangramento, $\mathrm{n}(\%)$ & $5(1,2)$ & $1(0,5)$ & $4(1,9)$ & 0,16 \\
Reação vasovagal, $\mathrm{n}(\%)$ & $11(2,7)$ & $4(2)$ & $7(3,4)$ & 0,38 \\
\hline
\end{tabular}

$\mathrm{n}$ = número de pacientes.

\section{LIMITAÇÃO DO ESTUDO}

O presente estudo apresentou como limitação o fato de ter sido realizado em um único laboratório de cardiologia intervencionista e de os procedimentos terem sido realizados por poucos operadores.

\section{CONCLUSÕES}

Pacientes submetidos a cateterismo cardíaco diagnóstico, utilizando introdutores arteriais $6 \mathrm{~F}$, podem deambular com segurança após três horas de repouso no leito. A redução do tempo de repouso traz maior conforto ao paciente, diminui o tempo de internação e contribui para aperfeiçoar os recursos existentes em relação à demanda crescente de exames.

\section{AGRADECIMENTOS}

Agradecemos a Romualdo Bolzani dos Santos e à equipe de enfermagem, pelo apoio e incentivo à realização deste estudo.

\section{CONFLITO DE INTERESSES}

As autoras declararam inexistência de conflito de interesses.

\section{REFERÊNCIAS BIBLIOGRÁFICAS}

1. Logemann $\mathrm{T}$, Luetmer $\mathrm{P}$, Kaliebe J, Olson K, Murdock DK. Two versus six hour of bed rest following left-sided cardiac catheterization and a meta-analysis of early ambulation trials. Am J Cardiol. 1999;84(4):486-8, A10.

2. Koch KT, Piek JJ, Winter RJ, Mulder K, Schotborgh CE, Tijssen JG, et al. Two hour ambulation after coronary angioplasty and stenting with $6 \mathrm{~F}$ guiding catheters and low dose heparin. Heart. 1999;81(1):53-6.

3. Keeling A, Taylor V, Nordt LA, Powers E, Fisher C. Reducing time in bed after cardiac catheterization (TIBS II). Am J Crit Care. 1996;5(4):277-81. Comment in: Am J Crit Care. 1996; 5(6):462.

4. Roebuck A, Jessop S, Turner R, Caplin JL. The safety of two-hour versus four hour bed rest after elective 6-French femoral cardiac catheterization. Coronary Health Care. 2000; (4):169-73 
Rocha VS, et al. Repouso de Três Horas Não Aumenta Complicações Após Cateterismo Cardíaco Diagnóstico com Introdutor Arterial 6 F: Ensaio Clínico Randomizado. Rev Bras Cardiol Invas. 2009;17(4):512-7.

5. Doyle BJ, Konz BA, Lennon RJ, Bresnahan FJ, Rihal SC, Ting $\mathrm{HH}$. Ambulation 1 hour after diagnostic cardiac catheterization: a prospective study of 1009 procedures. Mayo Clin Proc. 2006;81(12):1537-40. Comment in: Mayo Clin Proc. 2006; 81(12):1535-6.

6. Boztosun B, Gunes Y, Yildiz A, Bulut M, Saglam M, Kargin $\mathrm{R}$, et al. Early ambulation after diagnostic heart catheterization. Angiology. 2007;58(6):743-6.

7. Dumont CJ, Keeling AW, Bourguignon C, Sarembock IJ, Turner M. Predictors of vascular complications post diagnostic cardiac catheterization and percutaneous coronary interventions. Dimens Crit Care Nurs. 2006;25(3):137-42.

8. Brito Jr FS, Magalhães MA, Nascimento TCDC, Amorim
IMG, Almeida BO, Abizaid AC, et al. Incidência e preditores contemporâneos de complicações vasculares após intervenção coronária percutânea. Rev Bras Cardiol Invasiva. 2007;15(4):394-9.

9. Applegate RJ, Sacrinty MT, Kutcher MA, Baki TT, Gandhi SK, Kahl FR, et al. Vascular complications in women after catheterization and percutaneous coronary intervention 19982005. J Invasive Cardiol. 2007;19(9):369-74. Comment in: J Invasive Cardiol. 2007;19(9):375-6.

10. Juergens CP, Lo $S$, French JK, Leung DY. Vaso Vagal reactions during femoral arterial sheath removal after percutaneous coronary intervention and impact on cardiac events. Int J Cardiol. 2008;127(2):252-4. 